\title{
Virtual Screening and in Vitro Assay to Explore Novel Inhibitors from Black Pepper against Potential Targets of Radopholus Similis
}

\author{
Rosana O. Babu \\ Ph.D Research Scholar \\ Indian Institute of Spices \\ Research, Kozhikode, India
}

\author{
Krishna P. B. \\ Ph. D Research Scholar \\ Indian Institute of Spices \\ Research, Kozhikode, India
}

\author{
Santhosh J. Eapen, Ph.D \\ Principal Scientist \\ Indian Institute of Spices \\ Research, Kozhikode, India
}

\begin{abstract}
Radopholus similis, a migratory endoparasitic nematode that cause massive necrosis of plant tissues and destruction in host plants. The search for new nematode control molecules, particularly those of natural origin are of great urgency and importance. Phenylpropanoids belong to a major group of secondary metabolites produced by plants, mainly in defense response to biotic or abiotic stresses; literatures shows that chemical constituents of this family have bioactive compounds that can substitute current synthetic nematicides. But its efficacy and mode of action have not been demonstrated scientifically. In the present study, compounds from metabolic pathways of phenylpropanoid biosynthesis in black pepper (Piper nigrum L.) have been screened for nematicidal and potential target inhibiting activity towards burrowing nematode, $R$. similis and the mechanism of inhibition of novel targets has been studied with molecular docking. The 3D structures of phenylpropanoid biosynthesis related phytochemicals were used as ligands. Available eight novel target protein $(\beta-1,4$, endoglucanase, calreticulin-1, xylanase, cathepsin B-like cysteine proteinase, cathepsin Slike cysteine proteinase, cytochrome c-oxidase subunit III, glutathione S-transferase and transthyretin-like protein 3 precursor) of nematode associated with parasitic lifestyles and survival were selected as target molecule. Potential binding sites on each protein surfaces were predicted and screened phenylpropanoids have been docked to modeled targets of $R$. similis to assess their molecular interaction, binding energy and consequently their inhibitory activity. The docking results showed that thirteen phenylpropanoids possess similar dock score and hydrogen bond interactions, compared to current inhibitors and nematicides. Screening of these compounds in an in vitro assay showed that eight among the thirteen phenylpropanoids (syringaldehyde, salicylic acid, catechol, ferulic acid, coumaric acid, caffeic acid, tannic acid and Nvanillylnonanamide) caused maximum mortality to $R$. similis at $200 \mathrm{ppm}$. Ferulic acid at $250 \mathrm{ppm}$ and $500 \mathrm{ppm}$ reduced $R$. similis population in infected black pepper in green house study. Hence the compounds represent promising starting points as lead compounds of natural origin that inhibit $R$. simlis; this provides possibility to further exploit these compounds in nematode management. The study also helps in understanding various aspects of phenylpropanoid pathways that can be manipulated for in-situ production and enhancement of these compounds.
\end{abstract}

\section{Keywords}

Radopholus similis; burrowing nematode; black pepper; in vitro assay; phenylpropanoid pathway; molecular docking; molecular modeling; in silico prediction ; virtual screening.

\section{INTRODUCTION}

Radopholus similis (Cobb) Thorne is a migratory endoparasitic nematode moving through root tissues and destructively feeding on plant cells. As these nematodes cause extensive wounds in plant roots, secondary infection by bacteria and fungi is very common, that further damages the root system [1]. They can cause significant plant damage ranging from negligible injury to total destruction of plant material. The burrowing nematode, R. similis, attacks agronomic as well as horticultural crops and many weeds; it is reported to reproduce on more than 365 plant species $[2,3]$. $\mathrm{R}$. similis is probably the most widely distributed pest of Piper nigrum, Musa spp. and Citrus spp.; it also attacks many crops that are important in world commerce and in subsistence-type agriculture, a factor which makes it a significant agricultural pest [4]. Because of the severity of R. similis damage (particularly to black pepper, banana and citrus), extensive control programs have to be developed for prevention, to avoid economic crop loss. Parasitic helminths of plants and other organisms have a massive detrimental effect on agriculture. Many synthetic nematicides used currently are very unspecific and possess a high general toxicity so that they have been or will soon be banned by various governments. Alternative efficient methods to control nematode infestations are rare. Therefore, the search for new nematode control agents, particularly that of natural ones is of great economic interest [5]. The inhibition of potential targets essential for nematode survival has been extensively used methodology by several commonly employed drugs for destruction of glutathione S- transferase [6]. Nematicides like carbamate and organophosphorus causes acetylcholinesterase inhibition to inhibit nematode survival [7].

Understanding the molecular mechanisms of parasitism will help to develop novel therapeutics and preventative strategies. Although molecular biology has enabled rapid advances in elucidating the details of gene function in many tractable model organisms such as Caenorhabditis elegans, methods for the direct study of genes from parasitic nematodes have remained elusive. With more and more transferable technologies appearing, molecular studies of candidate genes and proteins that are broadly distributed in plant parasitic nematodes, will pave the way to unlocking the secrets of parasitism. Acquisition of novel genes associated with parasitic lifestyles and survival; and their neutralization can affect nematode survival, invasion, parasitism and growth, which can lead to an effective nematode control [8]. Identifying the potential targets, their molecular structure, function and interaction studies will help not only in understanding key enzymes required for the survival of nematodes; but will also help to understand various aspects in phenylpropanoid pathways that can be manipulated for increased production of these compounds for developing 
nematode resistant crops. Nematode resistant crops are generally considered the most favourable management option, as opposed to the much disputed use of chemical nematicides [9]. Nematicide mode of action refers to the lethal action of a nematicide on specific and vital life processes within tissues of the nematode. For example, broad spectrum fumigant nematicides penetrate the body wall of the nematode directly and do not have to be eaten to be effective [10]

Plants produce a wide range of biologically active chemicals, secondary metabolites, which are involved in plant defence against pest and diseases. The major classes of secondary metabolites include alkaloids, terpenoids and phenylpropanoids [11]. The bio-synthetic pathway of phenylpropanoids is well characterized and constitutes a potential target for improvement of resistance against nematodes [12]. Phenylpropanoid pathway is an important metabolic pathway responsible for the synthesis of a wide variety of secondary metabolic compounds. Phenylpropanoids belong to a collection of plant secondary metabolites derived from phenylalanine and have extensive diversity of functions as both structural and signaling molecules. The phenylpropanoid pathway is initiated by enzymatic conversion of phenylalanine to cinnamic acid, which is catalyzed by enzyme phenylalanine ammonia lyase (PAL) and is accountable for production of many plant specific natural products like caffeoylquinic acid isomers, coumarins, salicylic acid, flavonoids and isoflavonoids and lignin monomers. Many of plant-derived phenolic compounds (flavonoids, isoflavonoids, coumarins, and lignans) are secondary products of phenylpropanoid metabolism [13- 15]. Compounds synthesized from phenylpropanoid metabolism leads have an imperative role in plant defense responses to wounding and pathogen infection also in antioxidant activity and free radical scavenging properties [16- 17] chemical constituents of this family has bioactive compounds [18-19]. During last few years, much interest is there on natural and synthetic phenylpropanoids for medicinal use as antioxidant, UV screens, anticancer, anti-virus, anti-inflammatory, wound healing, and antibacterial agents [20]. Previous investigation on antimicrobial properties of phytochemicals from Piper species has revealed the accumulation of antimicrobial phenylpropanoids [21]. Nematode-antagonistic properties of phenylpropanoids on the behaviour of migratory endoparasites Radopholus similis and Pratylenchus penetrans and sedentary endoparasite Meloidogyne incognita has been studied by Wuyts et al., 2006; the study also assessed effects of phenylpropanoids on chemotaxis, motility, viability and hatch of nematodes. Wuyts et al., 2006 discovered that the resistant banana varieties had more phenylpropanoids than susceptible ones. Therefore, in present study a broad spectrum evaluation was made of the effects of black pepper phenylpropanoids, since there are several reports on chemical constituents of this family has bioactive compounds, antimicrobial compounds and nematicidal. Hence, compounds from phenylpropanoid metabolic pathway of black pepper have been screened for their action against burrowing nematode, R. similis through the interaction with potential targets.

In this study, much effort has been focused on determining inhibitory activity of plant-derived compounds from phenylpropanoid metabolic pathway of black pepper towards available potential targets of the burrowing nematode $\mathrm{R}$. similis. The study gives a better understanding of interaction between black pepper and its major nematode species Radopholus similis. Understanding interaction between plant parasitic nematodes and plant secondary metabolites, in particular phenylpropanoids helps to increase knowledge on plant defense against nematode. Better knowledge of resistance mechanisms in black pepper and of characteristic features of resistant varieties may provide rationale for genetic improvement; genetic manipulated for increased production of these compounds for developing nematode resistant crops. In this study both bioinformatics and cheminformatics tools have been used to model the potential targets of R. similis, and to screen phytochemicals to identify new bioactive molecules that may act as potent nematicide through action against potential targets of the organism. The study also present structural models of $\mathrm{R}$. similis potential targets using conventional molecular modeling techniques. Subsequently, cheminformatics-based drug design approach was followed to define the putative characteristic functional residues of each target to identify and characterize ligand binding sites of corresponding target. The results presented in this work represent a conceptual advance for comprehending the protein-ligand interactions and mechanism of action of nematode inhibitors. The screened and selected potential inhibitor candidates can then be further validated by molecular experiments to check their specificity against corresponding targets and their application in nematode management.

\section{Material and Methods}

\subsection{Potential targets of $R$. similis}

The novel targets of $R$. similis, involved in invasion, parasitism, growth and survival has been used in this study, which include all possible targets of $R$. similis based on the availability of sequence and template structure information for modeling, they are $\beta-1,4$, endoglucanase, calreticulin-1, xylanase, cathepsin B-like cysteine proteinase, cathepsin Slike cysteine proteinase, cytochrome c-oxidase subunit III, glutathione S-transferase and transthyretin-like protein 3 precursor. The selected targets and their importance in nematode survival are listed in Table 1.

\subsection{Molecular modelling of targets}

Since experimentally and computationally solved structures were not available for all the selected potential targets, molecular modeling was done by using Modeller9.10 software [22] and I-Tasser tool [23]. Target sequences were retrieved from NCBI database. Conserved motifs present in the selected template sequences were analyzed using Conserved Domain Database (CDD) search [24]. The sequences were then used to Blast all the protein sequences of available PDB structures. Template structures were downloaded from RCSB PDB (http://www.rcsb.org/pdb/home/home.do).

Table 1. Selected targets in $R$. similis (based on available sequence data) and their importance in nematode

\begin{tabular}{ll}
\hline $\begin{array}{l}\text { Target } \\
\text { from } \boldsymbol{R} \text {. similis }\end{array}$ & Importance in parasitism/ survival \\
\hline Calreticulin-1 & $\begin{array}{l}\text { Calreticulin/calregulin is a high-capacity } \\
\text { calcium-binding protein which is present in } \\
\text { most tissues of nematode, they have } \\
\text { multiple functions including regulation of } \\
\text { signalling and metabolic pathways and } \\
\text { controlling cell cycle progress [35- 37]. }\end{array}$ \\
& $\begin{array}{l}\text { They are cell wall degrading enzymes } \\
\text { which are necessary for nematodes during } \\
\text { invasion in to host plant and plays an }\end{array}$ \\
\hline$\beta-1,4$, & $\begin{array}{l}\text { important role in infection and parasitism of } \\
\text { plants [38- 44]. }\end{array}$ \\
& $\begin{array}{l}\text { They are capable of hydrolyzing substituted } \\
\text { xylan polymers into fragments of random }\end{array}$ \\
\hline Xylanase &
\end{tabular}




\begin{tabular}{|c|c|}
\hline & $\begin{array}{l}\text { size, functions of these enzymes are } \\
\text { associated with a variety of developmental } \\
\text { processes [45-49]. }\end{array}$ \\
\hline $\begin{array}{l}\text { Cathepsin B-like } \\
\text { cysteine proteinase }\end{array}$ & \multirow{2}{*}{$\begin{array}{l}\text { Cathepsin cysteine proteinases are involved } \\
\text { in a variety of important biological } \\
\text { processes and have been implicated in } \\
\text { molting, intestinal digestion and tissue } \\
\text { remodeling in free living and parasitic } \\
\text { nematodes; and is associated with larval } \\
\text { molting and cuticle and eggshell } \\
\text { remodeling [50-52]. }\end{array}$} \\
\hline $\begin{array}{l}\text { Cathepsin S-like } \\
\text { cysteine proteinase }\end{array}$ & \\
\hline $\begin{array}{l}\text { Cytochrome c } \\
\text { oxidase subunit III }\end{array}$ & $\begin{array}{l}\text { They controls the last step of food oxidation } \\
\text { and catalyze ATP synthesis [53-54]. }\end{array}$ \\
\hline $\begin{array}{l}\text { Glutathione S- } \\
\text { transferase }\end{array}$ & $\begin{array}{l}\text { Multifunctional dimeric enzymes involved } \\
\text { in the metabolization of a broad variety of } \\
\text { xenobiotics and reactive endogenous } \\
\text { compounds [55-58]. }\end{array}$ \\
\hline $\begin{array}{l}\text { Transthyretin-like } \\
\text { protein } 3 \text { precursor }\end{array}$ & $\begin{array}{l}\text { They are one of the most abundant } \\
\text { nematode-specific domains, nematode- } \\
\text { specific nature of this gene family makes it } \\
\text { a promising target for nematicides even } \\
\text { though their functional role is not correctly } \\
\text { identified [60-61]. }\end{array}$ \\
\hline
\end{tabular}

The side chains and hydrogen atoms were added for refining the structure and the stability of homology model has been validated by checking the geometry using PROCHECK [25]. The model was validated using Ramachandran plot [26]. Ramachandran plot was identified by Procheck program of Structural Analysis and Verification Server (http://nihserver.mbi.ucla.edu/SAVES/) and RAMPAGERamachandran Plot Analysis server (http://mordred.bioc.cam.ac.uk/ rapper/rampage.php). The statistics of non-bonded interactions between different atom types were detected and value of error function was analyzed by Verify_3D [27] and ERRAT [28] to identify overall quality factor of the model. Active site residues of modeled targets were predicted using CASTp server (http://stsfw.bioengr.uic.edu/castp/calculation.php).

\subsection{Ligand Structure}

The 3D structures of compounds were developed by ACD/ChemSketch Version 10.0 for Microsoft Windows using canonical smiles of the compounds collected from PubChem (http://pubchem.ncbi.nlm.nih.gov/). Energy minimization and molecular optimization of the compounds were done using Arguslab 4.0.1 [29]. Geometry optimization was carried out using AM1 (Austin Model 1), semi-empirical quantum mechanics force field in Arguslab4.0.1. The best conformer exhibiting lowest energy thus obtained based on energy minimization and geometry optimization were saved in *.pdb format for input into docking environment.

\subsection{Virtual screening \& Molecular docking}

The compounds of phenylpropanoid metabolic pathway from black pepper have been screened for activities such as antihelminthic (nematicidal) activity in PASS (Predicted Activity Spectrum of Small Molecules) server (http://www.pharmaexpert.ru/passonline/). PASS predicts over 4000 kinds of biological activity, including pharmacological effects, mechanisms of action, toxic and adverse effects, interaction with metabolic enzymes and transporters, influence on gene expression, etc., on the basis of structural formula of a substance [30]. PASS prediction gives out a list of activities with appropriate $\mathrm{Pa}$ and $\mathrm{Pi}$, sorted in descending order of difference $(\mathrm{Pa}-\mathrm{Pi})>0$. $\mathrm{Pa}$ (probability "to be active") estimates the chance that the studied compound is belonging to the sub-class of active compounds, $\mathrm{Pi}$ (probability "to be inactive") estimates the chance that the studied compound is belonging to the sub-class of inactive compounds based on resemblance with structures of the molecules, which are the most typical in a sub-set of "active and inactive" in PASS training set. For each type of activity of the biological activity spectrum; Pa-Pi values vary from 0.000 to 1.000 . As per PASS prediction of biological activity, if the value of $\mathrm{Pa}>0.5$ in a scale of 0 to 1 unit, the compound is expected to reveal the activity in experiments. If $\mathrm{Pa}>0.7$, the compound is very likely to exhibit the activity in wet lab experiments. The compounds which showed selected activity in PASS screening have been taken as ligands for the studies.

To further refine and validate the screened compounds, docking study was performed to identify interacting residues with the ligands, and to assess binding efficiency. Molecular docking study was carried out by using Molegro Virtual Docker [31]. MVD performs flexible ligand docking, so the optimal geometry of the ligand is determined during the docking. MVD includes MolDock Score [31] and PLANTS Score [32] for evaluating docking solutions. MVD returns multiple poses representing different potential binding modes. Clustering has been used to reduce the number of poses obtained during docking run and the most promising ones were taken. Molecules were prepared for docking; for which bonds, bond orders, explicit hydrogen, charges, flexible torsions, were assigned by the MVD program for both the protein and ligands. The intact protein structure was loaded on to MVD platform for docking process. Ignore distant atoms option is used to ignore atoms far away from the binding site. The search algorithm is taken as Moldock SE and numbers of runs are taken 10 and $\max$ iterations were 2000 with population size 50 and with an energy threshold of 100 also at each step least 'min' torsions/translations/rotations are tested and the one giving lowest energy is chosen. Pose clustering was done by tabu based clustering method, using this clustering technique each found solution is added to a 'tabu list': during the docking simulation the poses are compared to the ligands in this 'tabu list'. The Rerank Score uses a weighted combination of the terms used by the MolDock score mixed with a few addition terms (the Rerank Score includes the Steric (by LJ12-6) terms which are LennardJones approximations to the steric energy - the MolDock score uses a piecewise linear potential to approximate the steric energy) [33]. The reranking score function is computationally more expensive than the scoring function used during the docking simulation but it is generally better than the docking score function at determining the best pose among several poses originating from the same ligand. MolDock showed better overall performance in docking simulations when compared with other software.

For further refining the study, a comparative docking study was also performed with current potent nematicidecarbofuran, PubChem CID 2566 [34] to assess the efficiency of phenylpropanoids over synthetic inhibitor. Compounds with the least dock score and high interaction than carbofuran were taken for in vitro studies based on the availability of compound.

\subsection{In vitro \& In vivo assay}

All compounds were purchased from Sigma-Aldrich Chemical Co. Compounds were dissolved in aqueous DMSO $(1 \%)$ or distilled water and stock solutions $\left(10 \mathrm{mg} \mathrm{ml}^{-1}\right)$ were prepared and stored at $-20{ }^{\circ} \mathrm{C}$ after filter sterilization. 
Compounds were tested for nematode mortality in four concentrations $\left(200,100,50,20{\mu \mathrm{gml}^{-1}}^{2}\right)$ with three repetitions per concentration in a $96-w e l l$ plate. Controls were $1 \%$ DMSO and distilled water. A sample of 20-25 nematodes, consisting of adults and juveniles of $R$. similis, was taken in $50 \mu \mathrm{l}$ of water in each well, prior to the addition of the compound. Plates were then incubated for three days and the numbers of alive/dead nematodes were counted at every $24 \mathrm{~h}$ under a stereo zoom microscope. Finally, percentage of nematode mortality were calculated

Two concentrations of Ferulic acid $\left(250,500 \mu \mathrm{gml}^{-1}\right)$ were applied in infected black pepper. Five replicates per concentration were maintained. After 3 months, plant height, plant mortality \& nematode population were analyzed by taking 10 roots of $10 \mathrm{~cm}$ from each treatment and control (110 roots) in infected black pepper.

\section{RESULTS AND DISCUSSION \\ 3.1 Molecular modelling of targets}

The template sequences were confirmed with the presence of conserved domain corresponding to each target using CDD search. The structures with highest identity and sequence coverage were used as template to build the 3D structures of target proteins, details of template structures and percentage identity are listed in Supplementary file 1. Three dimensional structures of modeled protein, corresponding Ramachandran plot, ERRAT confidence value, overall quality factor of modeled proteins are displayed in Supplementary file 2 .

\subsection{Virtual screening \& Molecular docking}

PASS biological activity prediction for compounds in phenyl propanoid pathway revealed that 18 compounds out of 26 phytochemicals possess antihelminthic (nematicidal) activity based on predicted $\mathrm{Pa}$ and $\mathrm{Pi}$ values. PASS screening results for 18 compounds with predicted $\mathrm{Pa}$; $\mathrm{Pi}$ values for antihelmintic property is shown in Supplementary file 3.

To study the molecular basis of interactions and binding efficacy, screened compounds were docked with selected potential targets. Docking results for the ligands with each target is shown in Supplementary file 4. Docking of ligands with their highly interacting targets, binding energy scores, hydrogen bond interactions and interacting binding site residues were displayed in Table 2. Docking results showed that all 18 compounds docked satisfactorily to the potential targets with good (least) docking scores. Hence the phytochemicals with least MolDock Score, Rerank Score and greater number of hydrogen bond interactions were selected as promising lead compounds after docking studies. Out of 144 docked complexes (18 compounds docked with 8 potential targets) generated, potential hits were selected by
MolDock Score, Rerank Score and greater number of hydrogen bond interactions with binding site residues. Figure 1 displays the docking poses and interactions of best docked syringaldehyde- transthyretin complex.

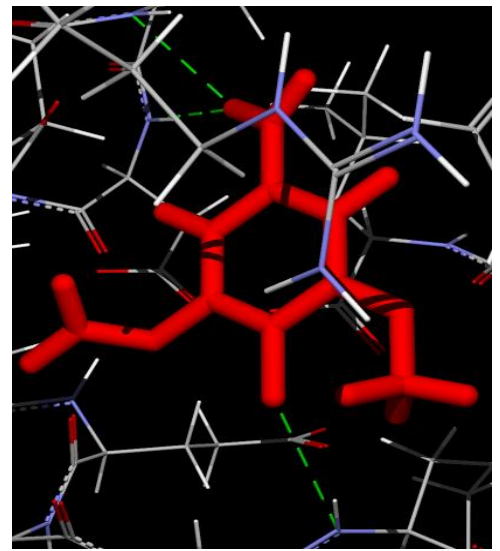

Figure 1. Syringaldehyde- transthyretin

The top hit ligand (syringaldehyde) targeted transthyretin like protein and established three hydrogen bonds with LEU49, CYS51, ASP58 which provide stability to the complex. From docking results it is evident that the compounds are interacting highly with 3 targets calreticulin1, GST and transthyretin like protein. Further observed best binding energy scores of the complex were, MolDock score: -183.38 , Rerank Score: -103.39 and Ligand efficiency: -6.78 , which suggested an energetically favorable interaction of syringaldehyde with transthyretin like protein. The residues involved in hydrogen bonding of docked complexes involved the binding site residues predicted by CASTp server.

For further refining the study, a comparative docking was performed with current potent nematicide- carbofuran, PubChem CID 2566 revealed that carbofuran also was interacting highly with the same 3 targets calreticulin1, GST and transthyretin like protein which had high interaction with phenylpropanoids. Phenylpropanoids which have least or similar binding energy scores, ligand efficiency and hydrogen bond interactions were confirmed with docking study. Binding energy scores and binding residues of nematode inhibitor- carbofuran is displayed in Table 3. Among the nematode inhibitors, currently nematicide- carbofuran markedly reduces worm viability [34]. The docking scores of Carbofuran are as follows: Moldock score: -130.59 , Rerank score of -129.45 , Ligand efficiency: -6.02 and 5 hydrogen bonds with transthyretin. Phytochemicals with least docking score than carbofuran are potential inhibitors of corresponding

Table 2. Phenylpropanoids with best docked target, corresponding binding energy scores and binding residues

\begin{tabular}{|c|c|c|c|c|c|c|}
\hline Compound & Target & $\begin{array}{l}\text { MolDock } \\
\text { Score }\end{array}$ & $\begin{array}{l}\text { Rerank } \\
\text { Score }\end{array}$ & $\begin{array}{l}\text { Ligand } \\
\text { efficiency }\end{array}$ & $\begin{array}{l}\text { No. of } \\
\text { Hbond }\end{array}$ & Binding Residues \\
\hline \multirow[t]{2}{*}{ syringaldehyde } & & -188.38 & -102.39 & -6.78 & 3 & \\
\hline & transthyretin & & & & & LEU49, CYS51, ASP58 \\
\hline ferulic acid & transthyretin & -183.63 & -103.81 & -5.62 & 6 & TYR138, ALA140, CYS51, VAL61, PHE87, LEU89 \\
\hline syringin & transthyretin & -178.48 & -123.74 & -6.71 & 11 & $\begin{array}{l}\text { LEU49, CYS51, ASP58, VAL61, PHE149, GLY151, ILE119, ILE121, } \\
\text { ILE123, TYR138, ALA140 }\end{array}$ \\
\hline salicylic acid & calreticulin & -159.64 & -113.15 & -2.31 & 3 & GLU390, ASP391, ASP391 \\
\hline $\begin{array}{l}\text { caffeoylquinic } \\
\text { acid }\end{array}$ & calreticulin & -144.01 & -103.36 & -4.13 & 12 & $\begin{array}{l}\text { ASP389, ASP389, GLU390, ASP391, ASP391, GLU392, ASP393, } \\
\text { GLU394, GLU396, GLU397, GLU398, LYS399 }\end{array}$ \\
\hline
\end{tabular}




\begin{tabular}{|c|c|c|c|c|c|c|}
\hline $\begin{array}{l}\mathrm{N}- \\
\text { vanillylnonanamide }\end{array}$ & calreticulin & -136.24 & -109.41 & -5.21 & 5 & GLU377, GLU378, GLU380, ASP391, GLU392 \\
\hline coniferin & calreticulin & -135.88 & -103.28 & -4.33 & 13 & $\begin{array}{l}\text { ASP389, GLU390, ASP391, GLU392, ASP393, GLU394, GLU396, } \\
\text { GLU397, GLU398, LYS399, ASP389, GLU390, ASP391 }\end{array}$ \\
\hline catechol & calreticulin & -135.87 & -98.44 & -2.74 & 6 & GLU390, ASP391, ASP391, GLU392, ASP393, GLU394 \\
\hline lantanilic acid & GST & -135.69 & -93.02 & -2.38 & 5 & GLY19, ARG25, PHE35, TYR176, TYR208 \\
\hline camaric acid & GST & -134.99 & -92.78 & -2.26 & 4 & GLY19, GLY18, PHE35, GLU36 \\
\hline scopolin & calreticulin & -132.85 & -104.18 & -4.16 & 13 & $\begin{array}{l}\text { ASP389, ASP389, GLU390, ASP391, GLU392, ASP393, GLU394, } \\
\text { GLU396, GLU397, GLU398, LYS399, GLU394, GLU396 }\end{array}$ \\
\hline caffeic acid & GST & -132.06 & -97.62 & -5.26 & 5 & ASP16, GLY18, GLY19, PHE35, GLU36 \\
\hline coumaric acid & calreticulin & -128.62 & -98.76 & -5.81 & 5 & GLU377, GLU378, GLU380, ASP391, GLU392 \\
\hline spermidine & calreticulin & -120.14 & -76.67 & -7.66 & 6 & GLU390, ASP391, ASP391, GLU392, ASP393, GLU394 \\
\hline synapic acid & GST & -112.32 & -92.64 & -4.53 & 5 & ASP16, GLY18, GLY19, PHE35, GLU36 \\
\hline synapaldehyde & calreticulin & -103.11 & -77.52 & -5.16 & 4 & GLU392, ASP393, GLU394, GLU396 \\
\hline cinnamic acid & GST & -98.43 & -66.99 & -5.18 & 2 & GLY19, GLY19 \\
\hline oleanolic acid & GST & -94.51 & -65.01 & -1.97 & 5 & GLY19, GLY18, PHE35, GLU36, ASP37 \\
\hline
\end{tabular}

Table 3. Binding energy scores and binding residues of nematode inhibitor- Carbofuran with $R$. similis targets

\begin{tabular}{llllll}
\hline Protein Name & $\begin{array}{l}\text { MolDock } \\
\text { Score }\end{array}$ & $\begin{array}{l}\text { Rerank } \\
\text { Score }\end{array}$ & $\begin{array}{l}\text { Ligand } \\
\text { efficiency }\end{array}$ & $\begin{array}{l}\text { No. of } \\
\text { Hbond }\end{array}$ & Binding Residues \\
\hline $\begin{array}{l}\text { Transthyretin-like protein } \\
3 \text { precursor }\end{array}$ & -130.59 & -129.45 & -6.02 & 5 & CYS51, CYS51, ASP58 \\
$\begin{array}{lllll}\text { Glutathione S-transferase } \\
\text { Calreticulin 1 }\end{array}$ & -118.19 & -70.64 & -4.55 & 4 & GLY176,GLY19, GLY19, \\
$\begin{array}{l}\text { Xylanase } \\
\begin{array}{l}\text { Cytochrome c oxidase } \\
\text { subunit III }\end{array}\end{array}$ & -112.07 & -85.04 & -4.31 & 2 & GLU392, ASP391 \\
$\begin{array}{l}\text { B-1, 4, endoglucanase } \\
\begin{array}{l}\text { Cathepsin B-like cysteine } \\
\text { proteinase }\end{array}\end{array}$ & -103.58 & -70.64 & -3.98 & 1 & LYS232, SER228, SER266 \\
$\begin{array}{l}\text { Cathepsin S-like cysteine } \\
\text { proteinase }\end{array}$ & -94.06 & -30.81 & -3.61 & 2 & SER230 \\
\hline
\end{tabular}

targets. The study reveal that most of the phenylpropanoids have least dockscore than carbofuran (-130.59) and more number of hydrogen bonds. Hence syringaldehyde (Moldock score -188.38, Rerank score: -102.39, Ligand efficiency: 6.781 and 3H-bond), ferulic acid (Moldock score: -183.63, Rerank score: -103.81, Ligand efficiency: -5.62 and $6 \mathrm{H}-$ bonds), syringin (Moldock score -178.48, Rerank score: 110.74, Ligand efficiency: -6.71 and 11 H-bond), salicylic acid (Moldock score -159.64, Rerank score: -113.15, Ligand efficiency: -2.31 and 3 H-bond), caffeoylquinic acid (Moldock score: -144.01, Rerank score: -103.36, Ligand efficiency: -4.13 and $12 \mathrm{H}$-bond), $\mathrm{N}$ - vanillylnonanamide (Moldock score: -136.24, Rerank score: -109.41, Ligand efficiency: -5.21 and $5 \mathrm{H}$-bond), coniferin (Moldock score: 135.88, Rerank score: -103.28 , Ligand efficiency: -4.33 and 13 H-bond), catechol (Moldock score: -135.87, Rerank score: -98.44, Ligand efficiency: -2.74 and $6 \mathrm{H}$-bond), lantanilic acid (Moldock score: -135.69, Rerank score: 93.05, Ligand efficiency: 2.38 and $5 \mathrm{H}$-bond), camaric acid (Moldock score: -134.99, Rerank score: -92.78 , Ligand efficiency: -2.26 and 4 H-bond), scopolin (Moldock score: -132.85, Rerank score: 104.18, Ligand efficiency: -4.16 and $11 \mathrm{H}$-bond), caffeic acid (Moldock score: -132.06, Rerank score: -97.62, Ligand efficiency: -5.26 and 5 H-bond) and coumaric acid (Moldock score: -128.62, Rerank score: -98.76, Ligand efficiency: -5.81 and $5 \mathrm{H}$-bond) which have least or similar binding energy scores, ligand efficiency and hydrogen bond interactions; hence these 13 compounds (syringaldehyde, ferulic acid, syringin, salicylic acid, caffeoylquinic acid, Nvanillylnonanamide, coniferin, catechol, lantanilic acid, camaric acid, scopolin, caffeic acid and coumaric acid) represent promising starting points as lead compounds for inhibiting $R$. similis novel targets and future development of strong nematicidal compounds. The results give a better knowledge of resistance mechanism or mechanism of phytochemical-mediated plant defense may provide rationale for genetic improvement; enhancing the productivity of these compounds for developing nematode resistant crops.

\subsection{In vitro \& In vivo assay}

The results of the docking study were validated through a direct contact in vitro assay. Based on the availability, eight compounds were taken for the in vitro assay studies. Nematode inhibition by eight phenolic compounds viz. syringaldehyde, salicylic acid, ferulic acid, catechol, coumaric acid, caffeic acid, tannic acid, $N$ - vanillylnonanamide and cinnamic acid at four different concentrations was evaluated in an in vitro assay on $R$. similis. Among the eight compounds, the maximum mortality was observed with the highest concentrations of syringaldehyde (100\%), salicylic 
acid (90), ferulic acid (71\%), catechol (71\%), coumaric acid $(65 \%)$, caffeic acid (48\%), tannic acid (30\%), Nvanillylnonanamide (28\%) and cinnamic acid (14\%). Mortality of Radopholus similis exposed to phenylpropanoids is shown in Table 4. Figure 2 Shows graph on mortality of $R$. similis (after 72 hours) exposure to selected Phenylpropanoids.

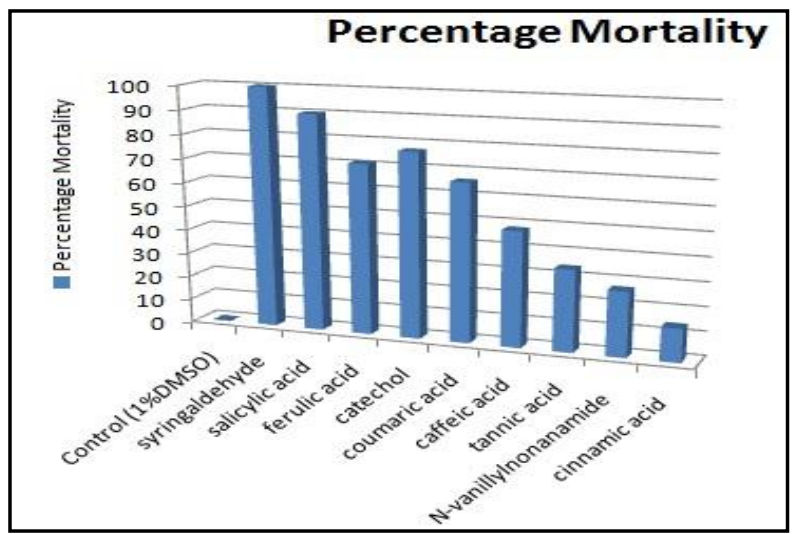

Figure 2. Graph shows mortality of $R$. similis (after 72 hours) exposure to selected Phenylpropanoids

In a similar study, Wuyts et al., 2006 have reported the efficacy of lignin related phenylpropanoids like ferulic acid, caffeic acid, coumaric acid etc. to be lethal to $R$. similis. As in this study, syringaldehyde and ferulic acid was the most potent motility inhibitor and nematicide for $R$. similis in the above study too. It was found to be repellent and highly nematistatic and nematicidal for $R$. similis and moderately active on $M$. incognita. Hydroxy cinnamic acids like ferulic acid are reported to occur as free phenolic acids or as glycoside-bound phenolic acids in plant roots. Banana cultivars have been correlated with constitutive levels of catechin, hydroxycinnamic acids, dopamine and constitutive and infection-induced levels of condensed tannins in their roots $[12,15]$. Results obtained from the in vitro assay confirmed the nematicidal potential of syringaldehyde, salicylic acid, ferulic acid, catechol, coumaric acid, caffeic acid, tannic acid, $N$ - vanillylnonanamide and cinnamic acid against $R$. similis.

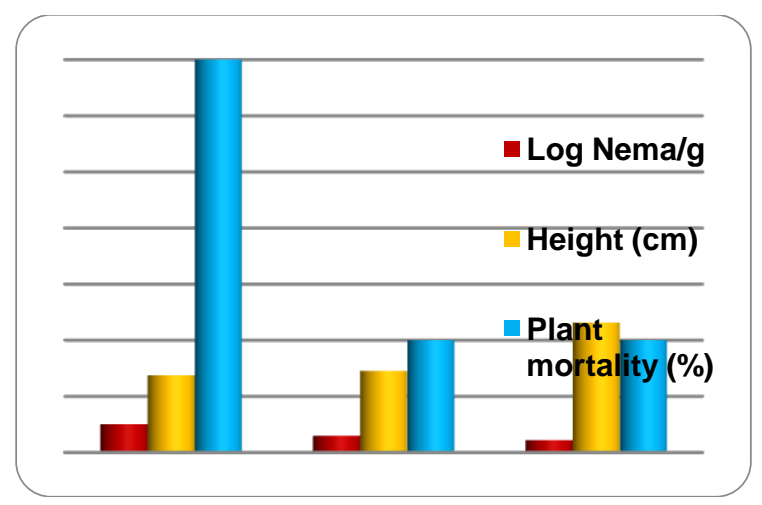

Figure 3. Shows the activity of ferulic acid at 250ppm and $500 p p m$ on reduction of $R$. similis population in infected black pepper rooted cuttings

Figure 3 Shows the activity of ferulic acid at 250ppm and $500 \mathrm{ppm}$ on reduction of $R$. similis population in infected black pepper in green house study. Ferulic acid was the only compound selected, because the compound is already reported in Wuyts et al., 2006 and it also shows efficacy in the above in silico study.

Table 4. Mortality of Radopholus similis exposed to phenylpropanoids

\begin{tabular}{lccc}
\hline Compound & \multicolumn{3}{c}{ In vitro assay (\% Mortality) } \\
\cline { 2 - 4 } & $\mathbf{2 4}$ hours & $\mathbf{4 8}$ hours & $\mathbf{7 2}$ hours \\
\hline Control (1\%DMSO) & $\mathbf{0}$ & $\mathbf{0}$ & $\mathbf{0}$ \\
syringaldehyde & 70 & 100 & 100 \\
salicylic acid & 80 & 80 & 90 \\
ferulic acid & 20 & 38 & 71 \\
catechol & 67 & 71 & 77 \\
coumaric acid & 21 & 38 & 66 \\
caffeic acid & 28 & 33 & 48 \\
tannic acid & 10 & 30 & 34 \\
N-vanillylnonanamide & 20 & 26 & 27 \\
cinnamic acid & 15 & 18 & 14 \\
\hline
\end{tabular}

\section{CONCLUSION}

To summarize, the study employed virtual screening protocol, molecular docking to identify novel inhibitors against potential targets of $R$. similis for better understanding of the functional mechanism of plant nematode interaction. The study focused on determining inhibitory activity of plantderived compounds from phenylpropanoid metabolic pathway of black pepper towards available potential targets of the burrowing nematode $R$. similis. The study gives a better understanding of interaction between black pepper and its major nematode species Radopholus similis. From the comparative docking study with current potent nematicidecarbofuran, the efficiency of phenylpropanoids over synthetic nematicide have been confirmed and reveals that 13 phenylpropanoids had least dockscore and more number of hydrogen bonds than carbofuran (Moldock score: -130.59 and 5 hydrogen bonds with transthyretin). The docking results also showed that carbofuran and phenylpropanoids were interacting highly with three potential targets viz. calreticulin1, GST and transthyretin like protein than the other targets. Screening of these compounds in an in vitro assay showed that eight among the thirteen phenylpropanoids (syringaldehyde, salicylic acid, catechol, ferulic acid, coumaric acid, caffeic acid, tannic acid and $\mathrm{N}$ vanillylnonanamide) caused maximum mortality to $R$. similis at $200 \mathrm{ppm}$. Ferulic acid at $250 \mathrm{ppm}$ and $500 \mathrm{ppm}$ reduced $R$. similis population in infected black pepper in green house study. Hence these 13 compounds (syringaldehyde, ferulic acid, syringin, salicylic acid, caffeoylquinic acid, Nvanillylnonanamide, coniferin, catechol, lantanilic acid, camaric acid, scopolin, caffeic acid and coumaric acid) with represent promising starting points as lead compounds of natural origin that inhibit $R$. simlis; this provides possibility to further exploit these compounds in nematode management. 
Wuyts et al., 2006 discovered that the resistant banana varieties had more phenylpropanoids than susceptible ones. Understanding interaction between plant parasitic nematodes and plant secondary metabolites, in particular phenylpropanoids helps to increase knowledge on plant defense against nematode. Better knowledge of resistance mechanisms in black pepper and of characteristic features of resistant varieties may provide rationale for genetic improvement; genetic manipulated for increased production of these compounds for developing nematode resistant crops. Both bioinformatics and cheminformatics tools have been used to model the potential targets of $R$. similis, and to screen phytochemicals to identify new bioactive molecules that may act as potent nematicide through action against potential targets of the organism. The study also present structural models of $R$. similis potential targets using conventional molecular modeling techniques. Subsequently, cheminformatics-based drug design approach was followed to define the putative characteristic functional residues of each target to identify and characterize ligand binding sites of corresponding target. After comparative modeling and structural refinement of potential targets, the general quality assessment indicated that the constructed models had acceptable profiles. The top hits identified by virtual screening and docking may serve as potential inhibitors of corresponding target essential for survival and parasitism of nematode after experimental validation. Further, the backbone structural scaffold of 13 lead compounds predicted in this study could serve as building blocks for the design and development of potential inhibitors for corresponding targets. The results presented in this work represent a conceptual advance for comprehending the protein-ligand interactions and mechanism of action of inhibitors. The screened and selected potential inhibitor candidates can then be further validated by molecular experiments to check their specificity against corresponding targets and their application in nematode management. Results obtained from the in-vitro assay confirmed the nematicidal potential of lead compounds obtained from In-silico docking. Ferulic acid at 250ppm and $500 \mathrm{ppm}$ reduced $R$. similis population in infected black pepper in green house study. Hence these compounds of phenylpropanoid metabolic pathway are novel, alternative inhibitors of natural origin for nematode management $(R$. similis) through inhibition of potential targets for nematode survival. The identified lead compounds of natural source can be an alternative measures over synthetic nematicides, as their mechanism of action are specific and less toxic. Understanding interaction between plant parasitic nematodes and plant secondary metabolites, in particular phenylpropanoids helps to increase knowledge on plant defense against nematode; further studies can be taken on engineering enhanced production of these compounds in black pepper to ward off the nematode menace.

\section{ACKNOWLEDGMENTS}

Our thanks to Director, Indian Institute of Spices Research (IISR), Kozhikode, for providing the facilities and Dept. of Biotechnology (BTISnet), New Delhi for the funding.

\section{REFERENCES}

[1]Zunke U. (1991). Observations on the invasion and endoparasitic behaviour of the root lesion nematode Pratylenchus penetrans. J. Nematol. 22:309-320.

[2] Brooks F.E. (2008). Burrowing Nematode. The Plant Health Instructor. DOI: 10.1094/PHI-I-2008-1020-01.
[3] Holdeman Q. L. (1986). The Burrowing Nematode Radopholus similis sensu lato. Nematology Publication, California Department of Food and Agriculture, Division of Plant Industry, Sacramento, CA, USA.

[4] O'Bannon J.H. (1977). Worldwide dissemination of Radopholus similis and its importance in crop production. J. Nematol. 9(1):16-25.

[5] Chitwood D. J. (2002). phytochemical based strategies for nematode control. Annu. Rev. Phytopathol. 40:221-49.

[6] Gupta S. Bhandari Y.P., Reddy M.V., Harinath B.C., Rathaur S. (2005). Setaria cervi: immunoprophylactic potential of glutathione-S-transferase against filarial parasite Brugia malayi. Exp Parasitol 109:252-255.

[7] D.J. Pree, J.L. Townshend, and D. E. Archibald (1989). carbamate and organophosphorus nematicides: acetylcholinesterase inhibition and effects on dispersal. Journal of nematology 21(4):483-489.

[8] Rosso M.N., Jones J.T., Abad P. (2009). RNAi and functional genomics in plant parasitic nematodes: tools and discoveries in the post-genomic era. Ann. Rev. Phytopathol 47:207-232.

[9] Wuyts N., Swennen R., De Waele D. (2006). Effects of plant phenylpropanoid pathway products and selected terpenoids and alkaloids on the behaviour of the plantparasitic nematodes Radopholus similis, Pratylenchus penetrans and Meloidogyne incognita. Nematology, 8(1, 89-101(13)

[10] Noling J. W. (2011). Movement and Toxicity of nematicides in the Plant Root Zone. ENY-041 (formerly RF-NG002), one of a series from the Department of Entomology and Nematology, Florida Cooperative Extension Service, Institute of Food and Agricultural Sciences, University of Florida.

[11] Chitwood D. J. (2003). "Nematicides," in Encyclopedia of Agrochemicals (3), pp. 1104-1115, John Wiley \& Sons, New York.

[12] Collingborn F.M.B., Gowen S.R. and Mueller-Harvey I. (2000). Investigations into the biochemical basis for nematode resistance in roots of three Musa cultivars in response to Radopholus similis infection. J. Agric. Food Chemistry 48: 5297-5301.

[13]Dixon R.A. and Paiva N.L. (1995). Stress-induced phenylpropanoid metabolism. Plant Cell 7: 1085- 1097.

[14]Douglas C.J. (1996). Phenylpropanoid metabolism and lignin biosynthesis: from weeds to trees. Trends Plant Sci. 1: 171-178.

[15] Valette C., Andary C., Geiger J.P., Sarah J.L. and Nicole M. (1998). Histochemical and cytochemical investigations of phenols in roots of banana infected by the burrowing nematode Radopholus similis. Phytopathology 88: 1141-1148.

[16] Hahlbrock K. and Scheel D. (1989). Physiological and molecular biology of phenylpropanoid metabolism. Annu. Rev. Plant Physiol. Plant MoI. Biol. 40: 347-369.

[17]Nicholson R.L. (1992). Phenolic compounds and their role in disease resistance. Annu. Rev. Phytopathol. 30: 369-389. 
[18]Jensen S., Hansen J. and Boll P.M. (1993). Lignans and neolignans from Piperaceae. Phytochemistry 33: 523530 .

[19]Parmar V.S., Jain S.C., Bisht K.S., Jain R., Taneja P., Jha A., Tyagi O.M., Prasad A.K., Wengel J., Olsen, C.E. and Boll P.M. (1997). Phytochemistry of genus Piper. Phytochemistry 46: 597-673.

[20]Korkina L.G. (2007). Phenylpropanoids as naturally occurring antioxidants: from plant defense to human health. Cell Mol. Biol. (Noisy-le-grand). 53 (1):15-25.

[21] Masuda T., Inazumi A., Yamada Y., Padolina W.G., Kikuzaki H. and Nakatani N. (1991). Antimicrobial phenylpropanoids from Piper sarmentosum. Phytochemistry 30: 3227-3228.

[22] Eswar N., Marti-Renom M. A., Webb B., Madhusudhan M. S., Eramian D., Shen M., Pieper U., Sali A. (2006). Comparative Protein Structure Modeling With MODELLER. Current Protocols in Bioinformatics, John Wiley \& Sons, Inc., Supplement 15, 5.6.1-5.6.30.

[23] Yang Z. (2008). I-TASSER server for protein 3D structure prediction. BMC Bioinformatics, 9:40.

[24] Marchler-Bauer A, Zheng C, Chitsaz F, Derbyshire MK, Geer LY, Geer RC, Gonzales NR, Gwadz M, Hurwitz DI, Lanczycki CJ, Lu F, Lu S, Marchler GH, Song JS, Thanki N, Yamashita RA, Zhang D, Bryant SH. (2013.) CDD: conserved domains and protein three-dimensional structure. Nucleic Acids Res. Jan 1;41(D1):D348-52.

[25] Laskowski R A. (2001). PDBsum: summaries and analyses of PDB structures. Nucleic Acids Res., 29, 221222.

[26] Rob W.W. Hooft, Chris Sander and Gerrit Vrien (1997). Objectively judging the quality of a protein structure from a Ramachandran plot. Comput. Appl Biosci. 13: 425

[27] Eisenberg D., Luthy R., Bowie JU. (1997). VERIFY3D: assessment of protein models with three-dimensional profiles. Methods Enzymol. 277:396-404

[28] Colovos C. and Yeates T.O. (1993). Verification of protein structures: Patterns of nonbonded atomic interactions. Protein Sci.2:1511-1519.

[29] Peng, C., Ayali, P.Y., Schlegel, H.B. and Frisch M.J. (1995). Using redundant internal coordinates to optimize equilibrium geometries and transition states. J. Comput. Chem. 16: 49-51.

[30] Filimonov D.A. and Poroikov V.V. (1996). PASS: Computerized prediction of biological activity spectra for chemical substances. Bioactive Compound Design. Possibilities for Industrial Use. BIOS Scientific, Oxford, pp. 47-56.

[31] Thomsen R. and Christensen M.H. (2006). MolDock: a new technique for high-accuracy molecular docking. $J$. Med. Chem. 49: 3315-3321.

[32] Korb O., Stutzle T. and Exner T.E. (2009). Empirical scoring functions for advanced protein-ligand docking with PLANTS. J. Chem. Inf. Model. 49: 84-96.

[33] Yang JM and Chen CC. (2004). GEMDOCK: a generic evolutionary method for molecular docking. Proteins: 55(2):288-304
[34] Meher H.C., Gajbhiya V.T., Singh G., Kamara A. \& Chawla G. (2010). Persistence and nematicidal efficacy of carbofuran, cadusa fos, phorate and triazophos in soil and uptake by chickpea and tomato crops under tropical conditions. J. Agric. Food Chem., 58: 1815-1822.

[35] Michalak M, Milner RE, Burns K, Opas M. (1992). Calreticulin. Biochem J. 1992 Aug 1;285 ( Pt 3):681- 92.

[36] Sheridan J.p., Miller A.J. and Perry R. N. (2004). Early responses of resistant and susceptible potato roots during invasion by the potato cyst nematode Globodera rostochiensis, Journal of Experimental Botany 55, 751760.

[37] Abad P, Favery B, Rosso MN, Castagnone-Sereno P. (2003). Root-knot nematode parasitism and host response: molecular basis of a sophisticated interaction. Molecular Plant Pathology 4, 217-224.

[38] Shibuya H. and Kikuchi T. (2008). Purification and characterization of recombinant endoglucanases from the pine wood nematode Bursaphelenchus xylophilus. Biosci. Biotechnol. Biochem. 72 (5): 1325-1332.

[39] Haegeman A., Jacob J., Vanholme B., Kyndt T. and Gheysen G., (2008). A family of GHF5 endo-1,4-betaglucanases in the migratory plant-parasitic nematode Radopholus similis. Plant Pathol. 57, 581-590.

[40] Goellner M., Wang X., and Davis E. L. (2001). Endo-b1,4-glucanase expression in compatible plant-nematode interactions. The Plant Cell 13:2241-2255.

[41] Wang X., Meyers D., Yan Y., Baum T., Smant G., Hussey R., and Davis E. (1999). In planta localization of a b-1,4-endoglucanase secreted by Heterodera glycines. Molecular Plant-Microbe Interactions 12:64-67.

[42] Bakhetia M., Urwin P. E., and Atkinson H. J. (2007). qPCR analysis and RNAi define pharyngeal gland cellexpressed genes of Heterodera glycines required for initial interactions with the host. Molecular PlantMicrobe Interactions 20:306-312.

[43] Chen Q., Rehman S., Smant G., and Jones J. T. (2005). Functional analysis of pathogenicity proteins of the potato cyst nematode Globodera rostochiensis using RNAi. Molecular Plant-Microbe Interactions 18:621625 .

[44] Rehman S., Butterbach P., Popeijus H., Overmars H., Davis E. L., Jones J. T., Goverse A., Bakker J., and Smant G. (2009). Identification and characterization of the most abundant cellulases in stylet secretions from Globodera rostochiensis. Phytopathology 99:194-202.

[45] Makedonka Mitreva-Dautova, Erwin Roze, Hein Overmars, Leo de Graaff, Arjen Schots, Johannes Helder, Aska Goverse, Jaap Bakker, and Geert Smant (2006.) A Symbiont-Independent Endo-1,4- $\beta$-Xylanase from the Plant-Parasitic Nematode Meloidogyne incognita. MPMI Vol. 19, No. 5, pp. 521-529.

[46] Banik M., Garrett T. P. J., and Fincher G. B. (1996). Molecular cloning of cDNAs encoding (1-4)-beta-xylan endohydrolases from the aleurone layer of germinated barley (Hordeum vulgare). Plant Mol. Biol. 31:11631172 .

[47] Bih F. Y., Wu S. S. H., Ratnayake C., Walling L. L., Nothnagel E. A., and Huang A. H. C. (1999). The 
predominant protein on the surface of maize pollen is an endoxylanase synthesized by a tapetum mRNA with a long 5' leader. J. Biol. Chem. 274:22884-22894.

[48] Chen N. J., and Paull R. E. (2003). Endoxylanase expressed during papaya fruit ripening: Purification, cloning and characterization. Funct. Plant Biol. 30:433441.

[49] Suzuki M., Kato A., Nagata N., and Komeda Y. (2002). A xylanase, AtXyn1, is predominantly expressed in vascular bundles, and four putative xylanase genes were identified in the Arabidopsis thaliana genome. Plant Cell Physiol. 43:759-767.

[50] Guiliano DB, Hong X, McKerrow JH, Blaxter ML Oksov Y, et al. (2004). A gene family of cathepsin L-like proteases of filarial nematodes are associated with larval molting and cuticle and eggshell remodeling. Mol Biochem Parasitol 136(2): 227-242.

[51] Sankale Shompole and Douglas P. Jasmer (2001) Cathepsin B-like Cysteine Proteases Confer Intestinal Cysteine Protease Activity in Haemonchus contort. Vol. 276, No. 4, Issue of January 26, pp. 2928-2934.

[52] Urwin P.E., Lilley C.J., McPherson M.J., Atkinson H.J. (1997). Characterization of two cDNAs encoding cysteine proteinases from the soybean cyst nematode Heterodera glycines. Parasitology 114:605-613.

[53] Calhoun M.W., Thomas J.W., Gennis R.B. (1994). The cytochrome oxidase superfamily of redox-driven proton pumps. Trends Biochem. Sci, 19, pp. 325-330 95026991.
[54] Jacob JE. (2009). A unique genetic code change in the mitochondrial genome of the parasitic nematode Radopholus similis. BMC Res Notes, 2009 Sep 24. PMID 19778425.

[55] Brophy P.M. and Barrett J (1990). Glutathione-Stransferase in helminths. Parasitology 100: 345-349.

[56] Brophy P.M. and Pritchard D.I. (1992). Immunity to helminths: ready to tip the biochemical balance. Parasitol.Today 8: 419-422.

[57] Ahmad R., Srivastava A.K. and Walter R.D. (2008a). Purification and biochemical characterization of cytosolic glutathione-S-transferase from filarial worms Setaria cervi. Comparative Biochemistry and Physiology, Part B 151: 237-245.

[58] Bhargavi R, Vishwakarma S, Murty US (2005). Modeling analysis of GST (glutathione-S-transferases) from Wuchereria bancrofti and Brugia malayi. Bioinform 1:25-27.

[59] Jacob J, Vanholme B, Haegeman A, Gheysen G (2007). Four transthyretin-like genes of the migratory plantparasitic nematode Radopholus similis: members of an extensive nematode-specific family. Gene. Nov 1; 402(12):9-19

[60] McCarter JP, Mitreva MD, Martin J, Dante M, Wylie T, et al. (2003). Analysis and functional classification of transcripts from the nematode Meloidogyne incognita. Genome Biology 4(4): R26.

[61] Palha JA (2002). Transthyretin as a thyroid hormone carrier: Function revisited. Clin Chem Lab Med 40: 1292-1300. 